九州大学学術情報リポジトリ

Kyushu University Institutional Repository

\title{
Formation of Zinc Protoporphyrin IX form Myoglobin with Pork Loin Extract
}

Ishikawa, Hiroya

Faculty of Agriculture, Graduate School, Kyushu University

Yoshihara, Miyuki

Faculty of Agriculture, Graduate School, Kyushu University

Baba, $\mathrm{Ai}$

Faculty of Agriculture, Graduate School, Kyushu University

Kawabuchi, Taiki

Faculty of Agriculture, Graduate School, Kyushu University

他

https://doi.org/10.5109/4716

出版情報：九州大学大学院農学研究院紀要. 51 (1)，pp.93-97，2006-02-01. Faculty of Agriculture， Kyushu University

バージョン :

権利関係 : 


\title{
Formation of Zinc Protoporphyrin IX from Myoglobin with Pork Loin Extract
}

\author{
Hiroya ISHIKAWA*, Miyuki YOSHIHARA, Ai BABA, Taiki KAWABUCHI, \\ Masahiko SATO ${ }^{1}$, Masahiro NUMATA ${ }^{1}$ and Kiyoshi MATSUMOTO
}

\author{
Laboratory of Food Analysis, Division of Food Biotechnology, Department of \\ Bioscience and Biotechnology, Faculty of Agriculture, Graduate School, \\ Kyushu University, Fukuoka, 812-8581, Japan \\ (Received October 27, 2005 and accepted November 16, 2005)
}

\begin{abstract}
The formation of zinc protoporphyrin IX (ZPP) with pork loin extract was determined by visible absorption and fluorescent spectral analyses. After the anaerobic incubation (in the dark at pH 5.5 and $30^{\circ} \mathrm{C}$ ) of the extract with metmyoglobin and $\mathrm{ZnCl}_{2}$, characteristic peaks in absorption and spectra were observed at 417,546 , and $584 \mathrm{~nm}$. In fluorescent spectra, a peak was observed at $589 \mathrm{~nm}$. Formed amounts of ZPP estimated from the fluorescent intensity at $589 \mathrm{~nm}$ was $15.5 \mathrm{nmol} / \mathrm{mL}$-pork extract. The difference in the formations of ZPP under aerobic and anaerobic incubations was not significant. The ZPP formation was significantly facilitated by the use of ATP. Zinc chelatase activity of loin extract was assayed with protoporphyrin IX and it was estimated as $42 \mathrm{mU} / \mathrm{mL}$-extract. The Fe-Zn substituting activity of the extract was assayed with myoglobin as a substrate. Under the assay conditions at $\mathrm{pH} 5.5$ and $40^{\circ} \mathrm{C}, \mathrm{ZPP}$ was markedly increased with increasing time by using oxymyoglobin reduced with ascorbate, while little increase was observed with metmyoglobin. The activity was estimated as $4.1 \mathrm{mU} / \mathrm{mL}$-extract with oxymyoglobin. At pH 5.5-7.0, higher activities were observed at lower $\mathrm{pH}$.
\end{abstract}

\section{INTRODUCTION}

Myoglobin is a main contributing factor to the color of meat and meat products, and therefore their sensory quality. Myoglobin is liable to oxidize during storage or heating (Sakata, 2000). Undesirable discoloration of meat is due to the oxidation of myoglobin (accumulation of brown metmyoglobin) (Renerre, 1990). Nitrite is usually added to cured-meat products to prevent the oxidation of myoglobin. However, nitrate has a possibility of risk for human health (Cassens et al., 1979, and Shahidi, 1991), and hence, there has been a growing demand for nitrite-free meat products and an intensified effort to develop a substitute for nitrite.

Recently, highly stable red pigment to light and heating has been found in Parma ham, a traditional dry cured ham of Parma (Italy) (Sakata, 2000). Parma ham is produced using only sea salt (from the Mediterranean Sea) without nitrate. The red pigment of Parma ham is easily extracted with $75 \%$ acetone, and the visible absorption spectral pattern is distinct from those of other known myoglobin derivatives (Sakata, 2000). The red pigment is gradually transformed from a myoglobin derivative into a thermostable non-protein heme complex (Moller et al., 2003). Wakamatsu et al. (2004a) has been identified that the red pigment as Zn-protoporphyrin IX (ZPP), in which the heme iron is substituted by zinc. In addition, they reported the formation behavior of ZPP with pork loin homogenates (Wakamatsu et al., 2004b).

The purpose of this paper is to clarify the ability of pork loin extract to form ZPP. The formation of ZPP with the loin extract was invetigate in detail, and then

${ }^{1}$ Itoham Foods Inc., 1-2-1, Kubogaoka, Moriya, Ibaraki, 302-0104, Japan

* Corresponding author (E-mail: ishikawa@agr.kyushu-u.ac.jp) determined the several factors affecting on the ZPP formation. In addition, we determined zinc chelatase activity of the loin extract with protoporphyrin IX as a substrate to clarify the mechanism of the ZPP formation from myoglobin. Wakamatsu et al. (2004b) also investigated the ZPP formation with pork loin extract, but zinc chelatase activity have not been estimated. Thus, this is the first report on zinc chelatase activity of the loin extract. In addition, the $\mathrm{Fe}-\mathrm{Zn}$ substituting activity, which is the combined activity of Fe-releasing from heme and $\mathrm{Zn}$-chelating into heme, was assayed. Then, we investigated some factors affecting on zinc chelatase and the Fe-Zn substituting activities to characterize the ability of the extract on the ZPP formation.

\section{MATERIALS AND METHODS}

\section{Materials}

Fresh pork loin (Landrace, 6 months old, $110-115 \mathrm{~kg}$ of body weight) sample was obtained from Itoham Foods Inc. (Ibaraki, Japan), and stored at $-40^{\circ} \mathrm{C}$ until use. Myoglobin (metmyoglobin) from horse skeletal muscle was purchased from Wako Pure Chemical Industries, Itd. (Osaka, Japan). Zn-protoporphyrin IX disodium salt was purchased from Aldrich Chem.Co. (Wisconsin, USA) and used as a standard of ZPP. Disposable $\mathrm{O}_{2}$ absorbing and $\mathrm{CO}_{2}$ generating agent and oxygen indicator tablet were purchased from Mitsubishi Gas Chemical Co., Inc. (Tokyo, Japan). All other chemicals used in this study were of analytical reagent grade.

\section{Preparation of pork loin extract}

Pork loin was homogenized with an equal volume of $50 \mathrm{mM}$ of sodium phosphate buffer (pH 5.5) containing $150 \mathrm{mM} \mathrm{NaCl}$. Each homogenization was performed at $10,000 \mathrm{rpm}$ for $5 \mathrm{~min}$ with polytron homogenizer (Kinematica, Inc. USA). Each homogenate was soni- 
cated (Branson Sonifier 250, Branson Ultrasonics Co., USA) at $200 \mathrm{~W}$ for $1 \mathrm{~min}$, and then centrifuged at $10,000 \times \mathrm{g}$ for $20 \mathrm{~min}$. Each supernatant (pork extract) was filtrated with the membrane filter $(0.45 \mu \mathrm{m})$ and used in the following study. The protein concentration was determined by Bio-Rad protein assay kit (Bio-Rad Laboratories, USA) using bovine serum albumin as a standard.

\section{Anaerobic incubation of sample solution}

Each pork extract $(2 \mathrm{~mL})$ was mixed with $1 \mathrm{~mL}$ of metmyoglobin solution (final conc. of $2 \mathrm{mg} / \mathrm{mL}$ ) in a $10 \mathrm{~mL}$-container. In the case with reduced-myoglobin (oxymyoglobin) as a substrate, reduced-myoglobin was prepared by the addition of sodium ascorbate ( $4 \mathrm{mg} / \mathrm{mg}-$ myoglobin) before the mixing with pork extract. Then, $100 \mu \mathrm{L}$ of $\mathrm{ZnCl}_{2}$ (final conc. of $250 \mu \mathrm{g} / \mathrm{mL}$ ) and $1.9 \mathrm{~mL}$ of $50 \mathrm{mM}$ sodium phosphate buffer were added. Each solution was put into the gas-impermeable container and kept in dark under anaerobic condition at $30^{\circ} \mathrm{C}$. The anaerobic condition (oxygen concentration in the container; $<0.1 \%$ ) was achieved by using the disposable $\mathrm{O}_{2}$ absorbing and $\mathrm{CO}_{2}$ generating agent and checked with an oxygen indicator tablet. After incubation, each solution was centrifuged at $3,000 \times \mathrm{g}$ for $20 \mathrm{~min}$. The supernatant containing myoglobin was applied to the visible absorption spectral analysis. On the other hand, water-insoluble red pigment (ZPP) was extracted from the precipitate with $75 \%$ acetone and applied to visible absorption and fluorescent spectral analyses.

\section{Visible absorption and fluorescent spectral analy-} ses

Visible absorption spectra of myoglobin and ZPP were measured at $400-700 \mathrm{~nm}$ by using a spectrophotometer with the photodiode array (Multispec 1500, Shimadzu Co., Ltd., Kyoto, Japan). Fluorescent spectra of ZPP were measured at 550-650 nm at $410 \mathrm{~nm}$ for excitation by using a fluorescence spectrophotometer (Model 5300, Shimadzu Co., Ltd., Kyoto, Japan). The formed amount of ZPP (nmol/mL-pork extract) after the storage was determined by using the standard curve for ZPP preparation $(0.08-1.6 \mu \mathrm{mol} / \mathrm{mL}-75 \%$ acetone, $\mathrm{r}$ $=0.999$ ).

\section{Assays of zinc chelatase and Fe-Zn substituting activities}

Assay of zinc chelatase activity was performed by modifying the fluorimetric method described by Goldin and Little (1969). Each pork extract (0.4 mL) was mixed with equal volume of $50 \mathrm{mM}$ sodium phosphate buffer containing $2.5 \mathrm{mg}$ of ATP, and then $100 \mu \mathrm{L}$ of $1 \mathrm{mM} \mathrm{ZnCl}_{2}$ and $100 \mu \mathrm{L}$ of $90 \mu \mathrm{M}$ protoporphyrin IX solution were added. The solutions were kept at $40^{\circ} \mathrm{C}$ in water bath under aerobic condition. On the other hand, the anaerobic incubation was performed by using the solution degassed with a vacuum pump, and the container of the solution was sealed and kept at $40^{\circ} \mathrm{C}$ in a water bath. After incubation, $3 \mathrm{~mL}$ of acetone was added to the solution and each solution was centrifuged at $3,000 \times \mathrm{g}$ for $20 \mathrm{~min}$. Formed red pigment (ZPP) in $75 \%$ acetone was measured by the fluorescent spectral analysis. In addition, $\mathrm{Fe}-\mathrm{Zn}$ substituting activity was assayed by using oxymyoglobin reduced by sodium ascorbate as a substrate in a similar manner as above.

Formed amount of ZPP (nmol/mL-pork extract) after the incubation was determined by fluorescent spectral analysis as described above. One unit of the activity (zinc chelatase activity and Fe-Zn substituting activity) is defined as the amount of enzyme that catalyzes the formation of one nmol of ZPP in one minute at $40^{\circ} \mathrm{C}$ under assay condition.

Statistical analysis. Results are presented as means \pm SD and analyzed using paired Student's t-test. All statistical analyses were performed using Stat View software version 5.0J (SAS institute Inc. USA).

\section{RESULTS AND DISCUSSION}

\section{ZPP formation with pork loin extract after the anaerobic incubation}

The ZPP formation with pork loin extract was determined by visible absorption and fluorescent spectral analyses. Each incubation was performed at $\mathrm{pH} 5.5$ because the ZPP formation occurrs in Parma ham, whose $\mathrm{pH}$ was reported as 5.7 (dry-cured ham; $\mathrm{pH}$ 5.5) by Adamsen et al. (2004). Fig. 1 shows the visible absorption and fluorescent spectra of red pigment (in $75 \%$ acetone extracts) formed after the incubation the loin extract. The incubation time was set at $168 \mathrm{~h}$, because very slow formation of ZPP was observed with loin extract. Characteristic peaks in absorption spectra were observed at 417,546 , and $584 \mathrm{~nm}$ in both samples. In fluorescent spectra (Ex; $410 \mathrm{~nm})$, a peak at $589 \mathrm{~nm}$ was observed. We confirmed that these spectral patterns coincide with that of ZPP preparation and the red pigment extracted from Parma ham (Data not shown). These results were in agreement with those of Wakamatsu et al. (2004a, b). Formed amounts of ZPP
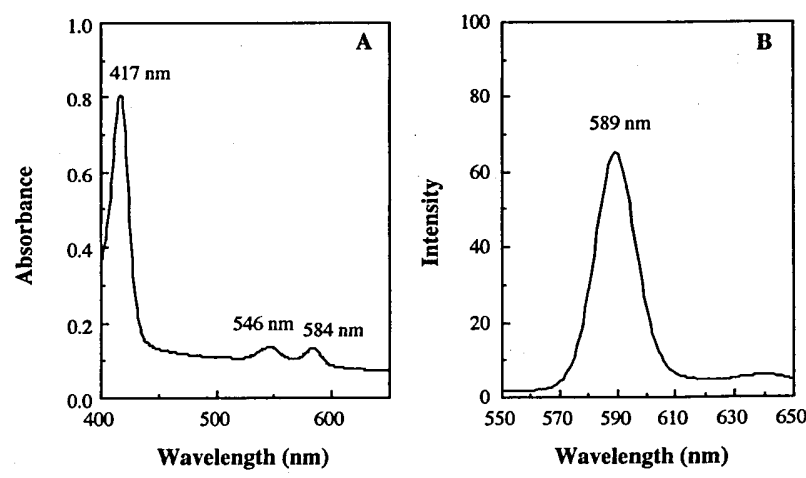

Fig. 1. The visible absorption (A) and fluorescent spectra (B) of red pigment (in $75 \%$ acetone) formed after the anaerobic incubation with pork loin extract. Each incubation was done in the dark at $\mathrm{pH} 5.5$ and $30^{\circ} \mathrm{C}$ with metmyoglobin and $\mathrm{ZnCl}_{2}$ (final conc.; $2 \mathrm{mg} / \mathrm{mL}$ and $250 \mu \mathrm{g} / \mathrm{mL}$, respectively). 
estimated from the fluorescent intensity at $589 \mathrm{~nm}$ was $15.5 \mathrm{nmol} / \mathrm{mL}-$ pork extract.

\section{ZPP formations with pork loin extract under the aerobic and anaerobic incubations}

The ZPP formation behaviors under anaerobic and aerobic conditions were compared. In both incubations, metmyoglobin was incubated with the loin extract and $\mathrm{ZnCl}_{2}$. The aerobic incubation was done without $\mathrm{O}_{2}$ absorbing and $\mathrm{CO}_{2}$ generating agent. Fig. 2 shows the formed amounts of ZPP after the anaerobic and aerobic incubations for $168 \mathrm{~h}$. In both incubations, similar intensity was observed, and aerobic incubation resulted in the formation of $95 \%$ of anaerobic incubation. These results suggested that anaerobic condition is not essential for the formation of ZPP. Wakamatsu et al. (2004b) studied the effects of oxygen on the formation of ZPP with pork loin homogenates. They concluded that oxygen inhibits the ZPP formation because this formation appeared to be minimal after aerobic incubation. However, the formation of ZPP was not appreciably inhibited by oxygen in our present study. Moreover, the reduction of metmyoglobin was also observed under aerobic incubation. After aerobic condition, the sample solution changed to bright red color and the reduction of metmyoglobin was confirmed by visible absorption spectra (Data not shown). Dean and Ball (1960) have been also reported that metmyoglobin is reduced during the aerobic storage by a natural metmyoglobin-reducing system in meat. Hence, oxygen would little affects the reduction of metmyoglobin. As described above, we assumed that zinc chelatase is responsible for the formation of ZPP. Zinc chelatase acts even under the aerobic condition (Nunn et al., 1988), and hence, the ZPP formation would be little affected by oxygen.

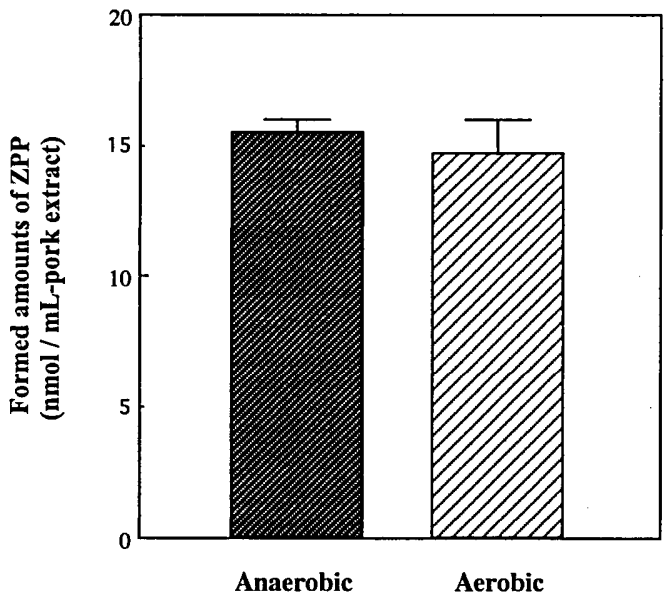

Fig. 2. Formed amounts of ZPP after the anaerobic and aerobic incubations. Both incubations were carried out as same in Fig. 1. Data represent the mean \pm SD $(n=5)$.

\section{The effect of ATP on the formation of ZPP with the loin extract}

The effect of ATP on the formation of ZPP was examined because zinc chelatase activity was increased by the addition of ATP (Goldin and Little, 1969). They demonstrated that ATP consistently stimulates the activity $1.5-2.5$ fold at the concentration of $2.5 \mathrm{mg} / \mathrm{mL}$-reaction mixture. In the present study, metmyoglobin was incubated with the extract and $\mathrm{ZnCl}_{2}$ in the dark at $30^{\circ} \mathrm{C}$ under anaerobic condition for $168 \mathrm{~h}$, and ATP was added to the incubating-sample at the concentration of $2.5 \mathrm{mg} / \mathrm{mL}$. Fig. 3 shows the formation of ZPP with and without ATP. As a result, the formation was significantly increased by the addition of ATP. This result suggested that zinc chelatase in heart extract could be activated by the addition of ATP. Goldin and Little (1969) suggested that Zn-ATP complex may serve as a more effective metal donor to form ZPP than free-Zn. However, Zn-ATP complex would not be formed under our assay condition at $\mathrm{pH} 5.5$, because ATP would be little ionized at the acidic $\mathrm{pH}$. Thus, the direct binding of ATP to zinc chelatase may stimulate the formation of ZPP.

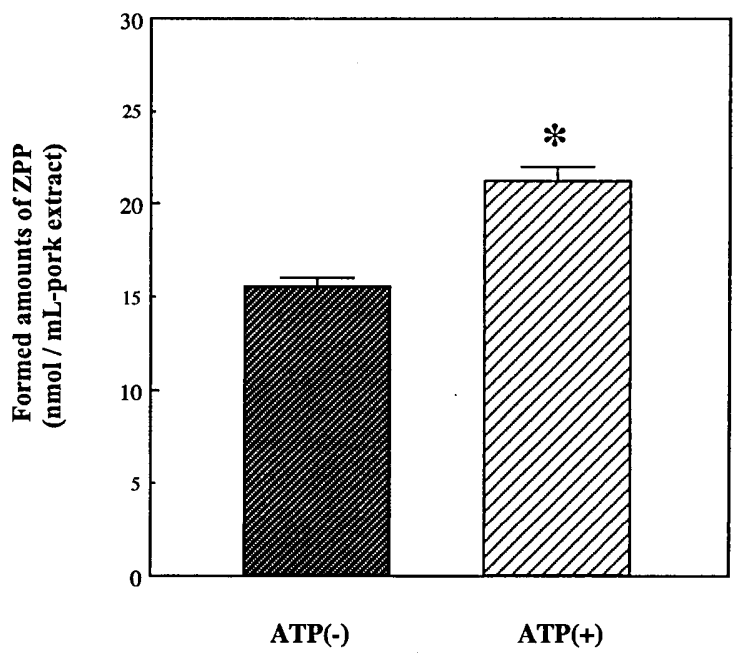

Fig. 3. The effect of ATP on the formation of ZPP by the anaerobic incubation. Data represent the mean $\pm S D$ $(\mathrm{n}=5) .{ }^{*} \mathrm{P}<0.01$ vs. ATP $(-)$.

\section{Zinc chelatase activity of the pork extract}

We determined zinc chelatase activity of pork loin extract with protoporphyrin IX as a substrate. Fig. 4 shows the formation behavior of ZPP during the aerobic incubation. ZPP was increased with increasing time, and linear ZPP formation was observed until $120 \mathrm{~min}$. Zinc chelatase activity was calculated from the slope of the linear curve $(r=1.000)$. The activity of the extract was estimated as $42 \mathrm{mU} / \mathrm{mL}$-extract (Fig. 5). In addition, the specific activity was estimated as $0.81 \mathrm{mU} / \mathrm{mg}$-protein. On the other hand, little formation of ZPP was observed with the heart extract treated by heating at $80^{\circ} \mathrm{C}$ and $15 \mathrm{~min}$ (Data not shown). This result suggested that nonenzymatically insertion of $\mathrm{Zn}$ into protoporphyrin IX would not occur under our 


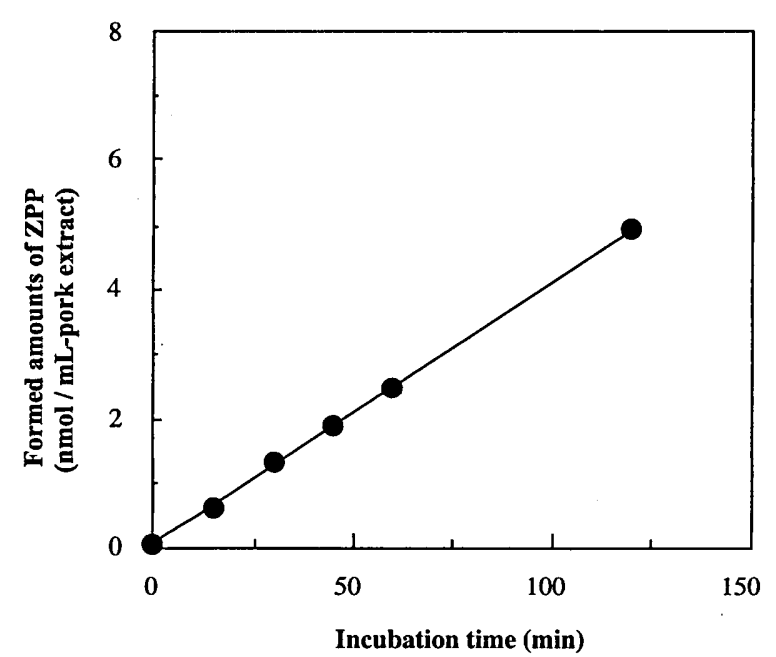

Fig. 4. The formation behavior of ZPP from protoporphyrin IX with the loin extract under the aerobic incubation.

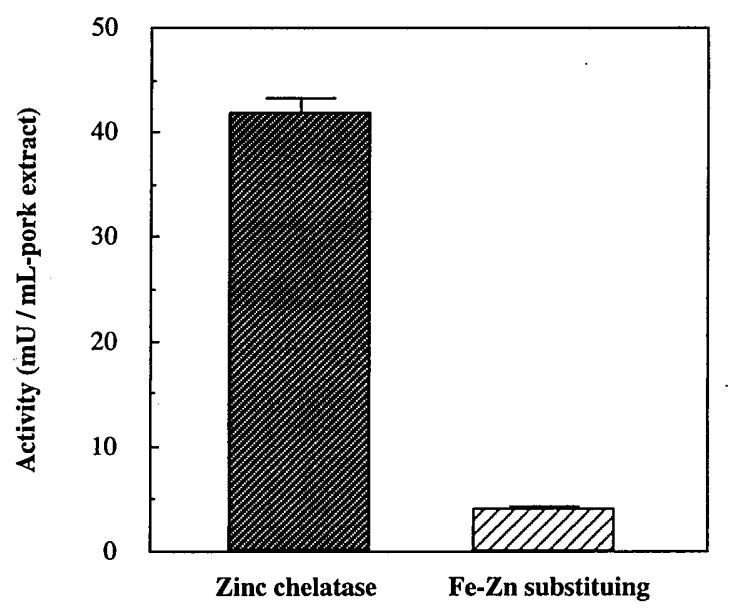

Fig. 5. Zinc chelatase and Fe-Zn substituting activities of the loin extract under aerobic condition. Data represent the mean $\pm S D(n=5)$.

experimental conditions. Wakamatsu et al. (2004b) was investigated the effects of pre-heating of pork loin homogenate on the ZPP formation from myoglobin. It has been shown that the ZPP formation was decreased with increasing of the pre-heating temperature, and no formation was observed at higher than $60^{\circ} \mathrm{C}$. They also suggested that the ZPP formation would be caused by the action of pork meat endogenous enzymes.

We examined the effect of oxygen on zinc chelatase activity. The activities under both conditions were nearly equal and there was no significant difference (Data not shown). Zinc chelatase from other source has been also assayed under aerobic condition (Nunn et al., 1988; Camadro and Labbe, 1982, and Goldin and Little, 1969). Their results showed that oxygen inhibits ferrochelatase activity, but does not inhibit zinc chelatase activity, and suggest that anaerobic condition is not an essential condition for the ZPP formation with zinc chelatase.

\section{The Fe-Zn substituting activity of the pork extract}

The Fe-Zn substituting activitiy of the pork extract was assayed with oxymyoglobin as a substrate. Oxymyoglobin was prepared by reducing metmyoglobin with sodium ascorbate ( $4 \mathrm{mg} / \mathrm{mg}$-myoglobin), and confirmed by the visible absorption analysis. Under the assay conditions at $\mathrm{pH} 5.5$ and $40^{\circ} \mathrm{C}$, the linear formation of ZPP was observed with oxymyoglobin and the activity was calculated from the slope of the linear formation curve, while little formation of ZPP was observed with metmyoglobin (data not shown). In the present assay system with oxymyoglobin, it was possible to estimated the activity of the loin extract within 6 hours, while 5 days-incubation was needed for the assay system by Wakamatsu et al. (2004b). The Fe-Zn substituting activity of the loin extract was shown in Fig. 5. The activity was estimated as $4.1 \mathrm{mU} / \mathrm{mL}$-extract with oxymyoglobin. The specific activity was 0.08 $\mathrm{mU} / \mathrm{mg}$-protein. The Fe-Zn substituting activity of the extract was $\sim 10$ times lower than their zinc chelatase activity. The release of $\mathrm{Fe}$ from heme would be rate-determining step on the ZPP formation from myoglobin.

\section{Effect of pH on zinc chelatase and Fe-Zn sub- stituting activities of the loin extract}

The effect of $\mathrm{pH}$ on zinc chelatase and $\mathrm{Fe}-\mathrm{Zn}$ substituting activities of pork extract were investigated. Fig. 6 shows these activities of the loin extract over the range of $\mathrm{pH} 5.5-7.0$. As a result, increase in zinc chelatase activity of the extract were observed at lower pH. At $\mathrm{pH} 5.5, \sim 1.4$ times higher activity was observed compared with that of at $\mathrm{pH} 7.0$. The optimum $\mathrm{pH}$ of zinc chelatase varies according to their origin. Zinc chelatase from barley (Goldin and Little, 1969) and yeast (Camadro and Labbe, 1982) have the optimum $\mathrm{pH}$ at 8.0 and 7.5, respectively. On the other hand, zinc chelatase of human lymphocytes has the optimum $\mathrm{pH}$ at 5.5 (Nunn et al., 1988). The activity profiles of the porcine extracts resemble their activity profile. Dailey et al. (1986) suggested that mammalian ferrochelatase
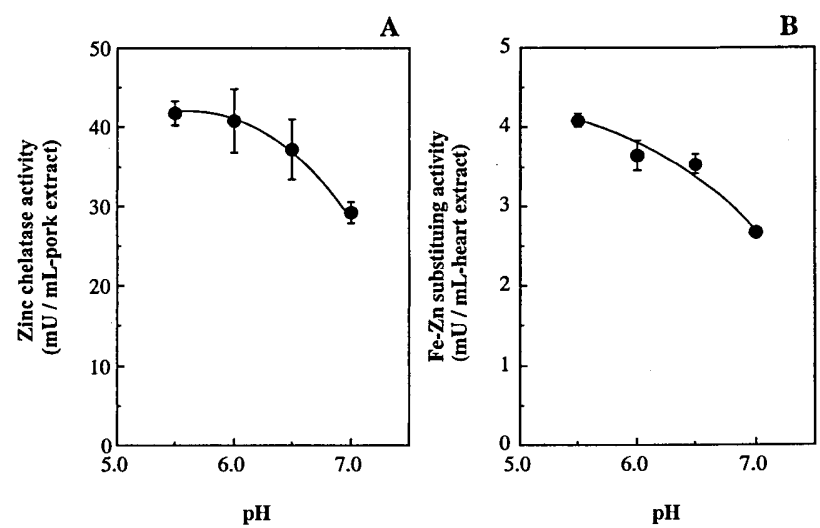

Fig. 6. The effect of $\mathrm{pH}$ on zinc chelatase activity (A) and Fe-Zn substituting activity (B) of the loin extract. Each incubation was carried out at $40^{\circ} \mathrm{C}$. Data represent the mean $\pm S D(n=3)$. 
shares many common characteristics. Similarly, mammalian zinc chelatase may exhibit similar activity profile on $\mathrm{pH}$. The Fe-Zn substituting activity of the extract also increased with the decrease in $\mathrm{pH}$, and the profiles were similar to those of zinc chelatase activity. Lower $\mathrm{pH}$ may also have effect on the release of iron from porphyrin ring of myoglobin.

\section{Conclusions}

The anaerobic incubation with pork loin extract resulted in the formation of ZPP. The formation was little affected by oxygen. The ZPP formation was stimulated in the presence of ATP. Zinc chelatase and Fe-Zn substituting activities were determined in the present study. The lower $\mathrm{pH}$ resulted in the higher activities. These activity profiles suggested that zinc chelatase would be closely related to ZPP formation.

\section{REFERENCES}

Adamsen, C. E., J. K. S. Moller, R. Hismani, and L. H. 2004 Skibsted Thermal and photochemical degradation of myoglobin pigments in relation to colour stability of sliced with nitrite salt. European Food Research and Technology, 218: $403-409$

Camadro, J. M, and P. Labbe 1982 Kinetic studies of ferrochelatase in yeast. Zinc or iron as competing substrate. Biochimica et Biophysica Acta, 707: 280-288

Cassens, R. G., M. L. Greaser, T. Ito, and M. Lee 1979 Reactions of nitrite in meat. Food Technology, 33(7): 46-57

Dailey, H., A., J., E. Fleming, and B. M. Harbin 1986 Purification and characterization of mammalian and chicken ferrochelatase. Method in Enzymology, 123: 401-408

Dean, R. W., and C. O. Ball 1960 Analysis of the myoglobin fractions on the surface of beef cuts. Food Technology, 14: 271-285

Goldin, B. R., and H. N. Little 1969 Metalloporphyrin chelatase from barley. Biochimica et Biophysica Acta, 171: 321-332

Moller, J. K. S., C. E. Adamsen, and L. H. Skibsted 2003 Spectral Characterisation of Red Pigment in Italian-type Dry-cured Ham. Increasing Lipophilicity during Processing and Maturation. Eur. Food Res. Technol., 216: 290-296

Nunn, A. V. W., P. Norris, J. L. M. Hawk, and T. M. Cox 1988 Zinc chelatase in human lymphocytes: Detection of the enzymatic defect in erythropoietic protoporphyria. Analitical Biochemistry, 174: 146-150

Renerre, M. 1990 Factors involved in the discoloration of beef meat. International Journal of Food Science and Technology, 25: 613-630

Sakata R., 2000 Studies on physicochemical characteristics of red pigments in meat products. Journal of Animal Science, 1: $1-16$

Shahidi, F. 1991 Developing alternative meat-curing systems. Trends in Food Science Technology, 2: 219-222

Wakamatsu, J., T. Nishimura, and A. 2004a Hattori A Zn-porphyrin complex contributes to bright red color in Parma ham. Meet Science, 67: 95-100

Wakamatsu, J., J. Okui, Y. Ikeda, T. Nishimura, and A. Hattori 2004b Establishment of a model experiment system to elucidate the mechanism by which $\mathrm{Zn}$-protoporphyrin IX is formed in nitrite-free dry-cured ham. Meat Science 68: $313-31$ 